\title{
On the influence of the sputtering in determining the resolution of a scanning ion microscope
}

\author{
V. Castaldo, ${ }^{\text {a) }}$ C. W. Hagen, and P. Kruit \\ Delft University of Technology, Lorentzweg 1, 2628 CJ Delft, the Netherlands \\ E. van Veldhoven and D. Maas \\ TNO Science and Industry, Stieltjesweg 1, 2628CK Delft, the Netherlands
}

(Received 8 July 2009; accepted 28 September 2009; published 7 December 2009)

\begin{abstract}
The determination of the quality of an imaging system is not an easy task for, in general, at least three parameters, strictly interdependent, concur in defining it: resolution, contrast, and signal-to-noise ratio. The definition of resolution itself in scanning microscopy is elusive and the case of scanning ion microscopy is complicated by the damage of the sample under the ion beam, which, especially for small features, can be the limiting factor. This is indeed the case for most focused ion beam systems, which exploit beams of $\mathrm{Ga}^{+}$. The only way to overcome this limit is to exploit sources of low mass ions, such as $\mathrm{H}^{+}$and $\mathrm{He}^{+}$. In this article the authors analyze the way the sputtering may affect the resolution, defined as smallest detectable feature in an image, of a scanning ion microscope, for heavy and light ions, in the case of spherical features. It appears that the fundamental limit to the resolution in scanning microscopy is not given by the spot size, but by the dynamics of the interaction of the beam with the sample and the consequent modification of the sample's geometry, even for beams of light ions. For example, in the case of Sn nanospheres under a $\mathrm{He}^{+}$beam, the authors found a minimum theoretical detectable particle size limit of $\sim 1 \mathrm{~nm}$ and an experimental limit of $\sim 5$ nm. () 2009 American Vacuum Society. [DOI: 10.1116/1.3253549]
\end{abstract}

\section{INTRODUCTION: MEANING AND INTERPRETATION OF RESOLUTION}

The notion of resolution is invoked whenever the quality of an imaging (or writing) tool has to be stated or advertised. ${ }^{1}$ To obtain scanning microscope images with a high resolution, the attributes of the sample are equally important as is the resolving power of the scanning microscope. Consequently, the ultimate resolving power of an instrument is not easily demonstrated, as the attributes of the average sample are often not fit for imaging at extreme magnification. In addition, the meaning of resolution is actually often unclear (and hence misinterpreted), as many different definitions and criteria coexist. Therefore it is difficult, if not impossible, to propose a single number that serves as ultimate judgment for tools that become more complex and multifunctional everyday. The main reason is that the ability of resolving small details in an image (what is commonly referred to as resolution) strongly depends on parameters that are not included in the traditional definitions: signal-to-noise ratio (SNR), contrast, and sample composition. ${ }^{2,3}$ Moreover, the case of resolution in scanning ion microscopy is complicated by yet another factor: The imaged sample undergoes more or less strong geometrical and compositional modifications due to the high momentum that is carried, and eventually transferred, by the impinging ions. In this case, not only the same machine can perform differently with different observed materials (which might happen also with electron microscopes because of different electron/atom interactions and more or less clean vacuum) but even the same material in a

${ }^{a)}$ Electronic mail: v.castaldo@tudelft.nl different geometrical structure can shift the balance between signal extraction and sample modification. A good review of this topic is contained in Ref. 4. [For a more complex definition of resolution, see Sato and Orloff in Ref. 5.]

In the present article some experimental results are presented and discussed, together with some preliminary simulation results, in order to make a further step toward a deeper understanding of the imaging performance of scanning ion microscopes (SIMs). The major conclusion is that for every specific sample a dose for optimal image acquisition exists. At that specific dose the sample modification uncertainty $\left(U_{S M}\right)$, which increases with longer exposures, is optimally balanced against the information uncertainty $\left(U_{I}\right)$, which obviously decreases when recording longer. [These two different and competing "uncertainties" were first introduced by the authors in Ref. 6 under the names of sputtering uncertainty and information uncertainty; here a broader concept is proposed, $U_{S M}$, that is more general than $U_{S}$ for it refers to any kind of sample modification (sputtering, contamination, and redeposition), and not only to the removal of sample material; $U_{S}$ is used in this article when referring to pure sputtering effect.]

\section{SPUTTERING AND IMAGING PERFORMANCE}

Most definitions of resolution in microbeam machines refer to the beam size at the sample. This works reasonably well at intermediate resolution for scanning electron microscopes (SEMs). However, they systematically ignore the destructive effect that a beam of massive particles, such as ions in SIMs or fast electrons in scanning transmission electron microscopy, can have on the sample: Details might have dis- 


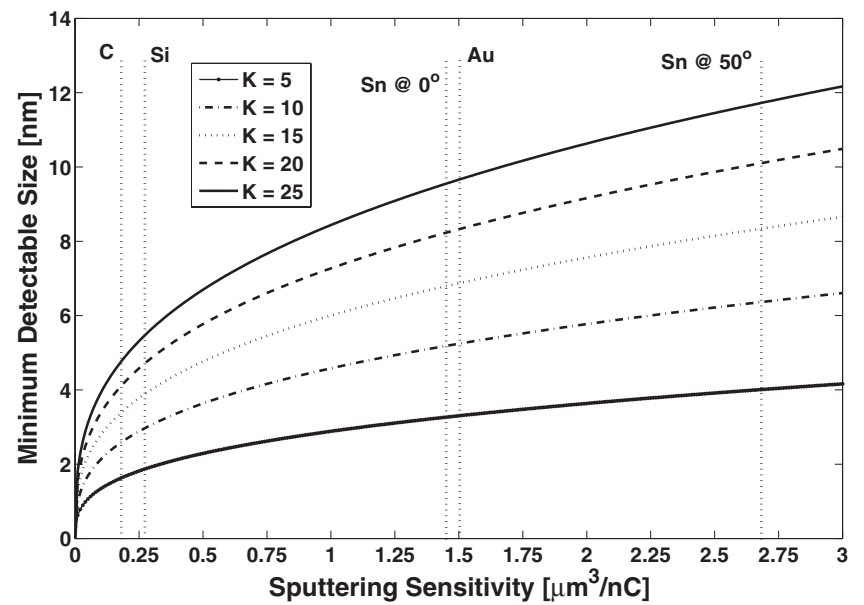

FIG. 1. Minimum detectable feature size in a SIM from Eq. (1), as a function of sputtering sensitivity $S$ at different values of $K$, for $\delta=2$ and $\Omega=0.5$. In the graph the sputtering sensitivity of different target materials for $30 \mathrm{keV}$ $\mathrm{Ga}^{+}$ions is highlighted.

appeared before they can be seen against the noise in the image. Orloff ${ }^{7}$ and Orloff $e t a l^{3}$ addressed the issue, proposing the following equation for the size of the smallest detectable particle $\left(D_{\min }\right)$ in a focused ion beam (FIB):

$$
D_{\min }=\sqrt[3]{\frac{e S K^{2}(1+\delta)}{\Omega^{2} \delta}},
$$

where $K$ is the SNR, $\delta$ (secondary electrons/primary ion) is the secondary electron (SE) yield, $\Omega$ (scan step size/beam diameter) is a measure of the overlap, and $S\left(\mu \mathrm{m}^{3} / \mathrm{nC}\right)$ is the sputtering sensitivity, defined as

$$
S=\frac{Y A}{\rho N_{0} e},
$$

where $\rho$ and $A$ are the target density and the atomic weight, respectively, $N_{0}$ is Avogadro's number $\left(6.02 \times 10^{23}\right.$ at $\left./ \mathrm{mol}\right)$, and $Y$ is the sputter yield (sputtered atoms/primary ion). It can be more practical to express $D_{\min }$ as a function of scanning time $t_{\text {scan }}$ instead of SNR, ${ }^{8}$

$$
D_{\min }=\sqrt[3]{\frac{S}{\Omega^{2}} \frac{I_{\text {beam }}}{p_{x}} t_{\text {scan }}},
$$

where $I_{\text {beam }}$ is the ion current and $p_{x}$ is the total number of pixels in the image. Figure 1 shows $D_{\min }$ as a function of the sputtering sensitivity $S$ for different levels of SNR in the image.

The conclusion is that for features in the order of a few nanometers, the resolution, defined as the smallest observable particle, is actually determined by the competition between sputtering and SE production/collection, at least for heavy ions like $\mathrm{Ga}^{+}$(atomic weight: 69.723). Similar to the limit posed by sputtering, other effects that damage the sample while imaging (e.g., contamination, heating, and charging, to list some of the sample damaging factors that occur in both electron and ion microscopies) also contribute to raising the lowest limit for detectable particles. Equation

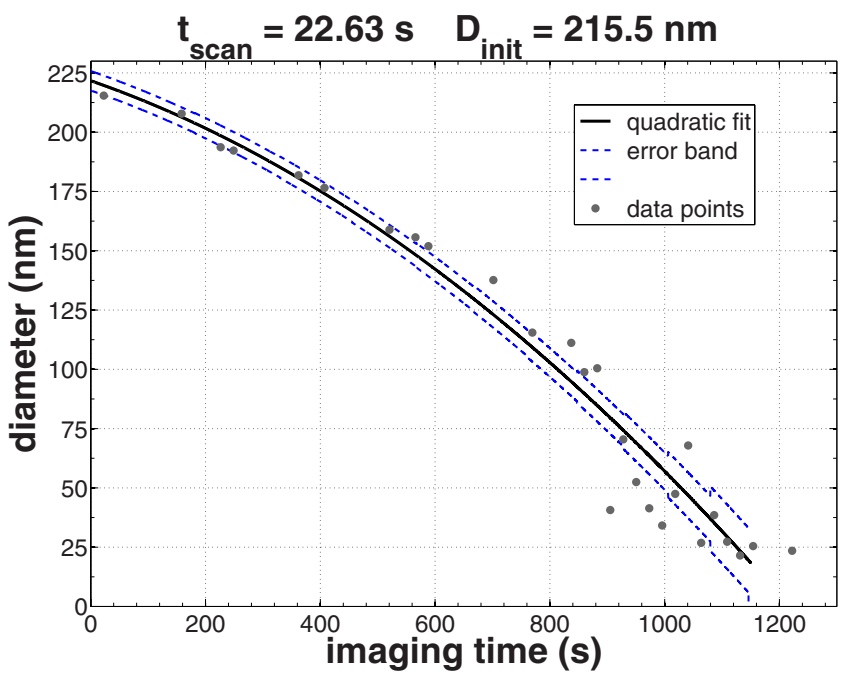

FIG. 2. (Color online) Size of a Sn ball plotted vs the imaging time with $\mathrm{Ga}^{+}$ ions at $30 \mathrm{keV}$.

(1) represents a fundamental limit to the imaging performance of a FIB. Even if the predicted resolution is hardly achieved in practice, as it has been stated under the assumptions of perfect collection of secondaries and Poissondistributed primary beam and ejected SEs, it is important in the sense that it establishes a theoretical limit.

A semiempirical method for evaluating the performance of a FIB imaging machine was proposed by Castaldo et al. in Ref. 8. The underlying idea is quite simple: A feature of a given size on the sample is imaged over and over until it fully disappears. The evolution of the feature size versus the scanning time (or ion dose, to normalize for different values of the ion current), as shown in Fig. 2, together with the relative error connected to each measurement, contains actually a lot of information. There are two competing factors that determine the resolution: the removal of sample material (or, more in general, the modification of the sample) that gives rise to $U_{S M}$, and the collection of information from the sample, which leads to $U_{I}$. Clearly, by increasing the scanning time the collected information increases but so does the sample modification by, e.g., sputtering: $U_{S M}$ and $U_{I}$ move in opposite directions. In terms of "size versus time" curve, this means that increasing the scanning time the error band $\left(U_{I}\right)$ at each point narrows, but the distance between two subsequent measuring points (connected to $U_{S M}$ ) increases. If these two quantities are plotted versus the scanning time, there will be a point in which the two curves intersect: For longer scanning time $U_{S M}$ is the limiting factor, for shorter scanning times the resolution is limited by the noise in the image. This point represents in fact the optimum imaging condition for the machine and the specific sample: It represents the optimal scanning time (or optimal imaging dose), i.e., the scanning time for which the smallest detectable particles are visualized.

Despite its simplicity, the size versus time curve tells even more than that. Consider two different microscope users, one working in the $3 \mathrm{D}$ integrated circuit (IC) industry and one 


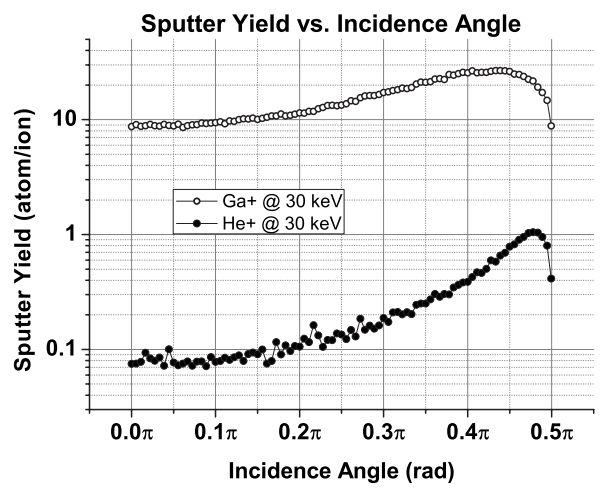

FIG. 3. Sputter yield vs incidence angle as obtained from TRIM simulation for $\mathrm{Ga}^{+}$and $\mathrm{He}^{+}$at $30 \mathrm{keV}$.

studying deposition mechanisms at the nanoscale. The first one will be interested in inspecting microchips and in precisely measuring the size of the features, while the second one will try to look at the smallest possible details. Both of them will look for high resolution, but in practice the two needs are two different ones. This distinction is clearly visible on the size versus time curve. The smallest detail can be determined from the right side: When the semiamplitude of the error band comes to coincide with the size of the observed feature, the limit of the resolution is reached, for a smaller detail cannot be distinguished anymore from the background noise. However when it comes to the precision of the measurement, things are different. Suppose we want to know the size of a line, we can image it with a FIB, many times, and build up a size versus time curve together with the error band. The curve can then be fitted and the original size can be recovered tracing back to imaging time of 0 . The error connected with this measurements is thus given by the amplitude of the band on the left side of the curve and it is generally smaller. Of course, these two concepts coincide when the initial size of the feature is small enough to be already on (or below) the limit of detection, in which case there will be only one point in the curve. However, in general, these two concepts must be kept separated and a curve like the one in Fig. 2 is a good reminder of that.

\section{GA+ VERSUS HE+: SIMULATIONS AND FIRST RESULTS}

The first step in an attempt to evaluate the possibilities of a FIB exploiting light ions, such as $\mathrm{He}^{+}$(atomic weight: 4.003 ), is thus to calculate what the lowest theoretically achievable resolution is. This can be done easily, for example, for $\mathrm{Sn}$ as target material, which has been used in the experiments discussed in this article. Figure 3 shows the sputter yields for $\mathrm{Ga}^{+}$and $\mathrm{He}^{+}$on $\mathrm{Sn}$, at $30 \mathrm{keV}$, as obtained by Monte Carlo calculation with the popular code TRIM, freely distributed by Ziegler. ${ }^{8}$ Eqs. (1) and (2) do not consider the dependence of $Y$ on the incidence angle; a mean value $\bar{Y}$ can thus be used. Table I shows the results obtained for $\delta=2$ and $\Omega=0.5$.
TABLE I. Sputtering sensitivities and minimum detectable features as obtained from Eqs. (2) and (1) for Sn target and beams of $\mathrm{Ga}^{+}$and $\mathrm{He}^{+}$at 30 $\mathrm{keV}$.

\begin{tabular}{lcccc}
\hline \hline & $\begin{array}{c}\bar{Y} \\
(\mathrm{at} / \mathrm{ion})\end{array}$ & $\begin{array}{c}S \\
\left(\mu \mathrm{m}^{3} / \mathrm{nC}\right)\end{array}$ & $\begin{array}{c}D_{\text {min }}^{K=5} \\
(\mathrm{~nm})\end{array}$ & $\begin{array}{c}D_{\text {min }}^{K=25} \\
(\mathrm{~nm})\end{array}$ \\
\hline $\mathrm{Ga}^{+}$ & 15.5 & 2.62 & 4.00 & 11.6 \\
$\mathrm{He}^{+}$ & 0.26 & 0.044 & 1.02 & 2.98 \\
\hline \hline
\end{tabular}

This is an important result, showing that using $\mathrm{He}^{+}$ions the resolution in a FIB imaging system can never go below $\sim 1 \mathrm{~nm}$, a value that is reached, in theory, only at the minimum acceptable level of the SNR. In any condition of noise, anyway, a $\mathrm{He}^{+}$beam allows for a resolution about four times higher than the one achievable using $\mathrm{a} \mathrm{Ga}^{+}$beam in the same conditions.

At this point it is interesting to see what happens to a more realistic system under different sputtering conditions. Starting again from the simulated sputtering yields as a function of incidence angle, the shrinking of $\mathrm{Sn}$ balls under irradiation with a $\mathrm{Ga}^{+}$beam and a $\mathrm{He}^{+}$beam at $30 \mathrm{keV}$ has been simulated. The model is rather simple, based on a continuum approach and not taking into account the single ion/atom interactions (for the details of the simulation, see Ref. 8). The result is shown in Fig. 4: The two simulations have been carried out for a Sn ball of initial diameter $130 \mathrm{~nm}$ and the size is plotted against the ion dose so that curves obtained for different ion currents, dwell times, and magnifications can be directly compared. The scale on the $x$ axis is logarithmic since the doses needed for an appreciable diameter reduction are rather different. What appears is that while the ratio between the average sputter yields is about 60 (from Table I, $\left.\bar{Y}_{\mathrm{GaSn}}^{30 \mathrm{keV}} / \bar{Y}_{\mathrm{HeSn}}^{30 \mathrm{keV}}=59.6\right)$, the ratio of the dose required to totally destroy the ball is about 100 , meaning that the different sputtering rates are somehow amplified when it comes to the modification of small features. [Simply averaging the sputter rates is probably not the right metric for typical threedimensional (3D) objects such as $\mathrm{Sn}$ balls. A cleaner experiment would be studying sputtering damage to flat twodimensional objects, such as lines.] Figure 5 shows the same curves but this time based on experimental results: A stan-

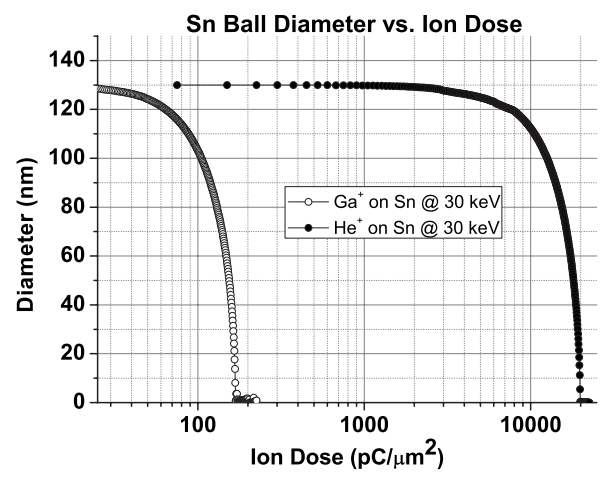

FIG. 4. Simulation of the shrinking of a Sn ball of initial diameter $130 \mathrm{~nm}$ under irradiation with $\mathrm{Ga}^{+}$and $\mathrm{He}^{+}$at $30 \mathrm{keV}$. 


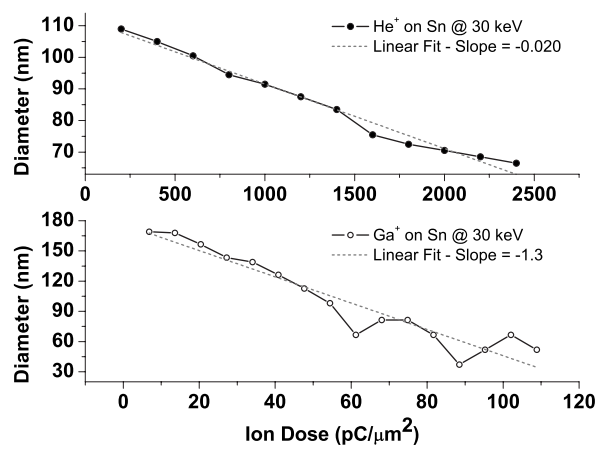

Fig. 5. Measurement of the shrinking of two Sn balls of similar initial diameter under irradiation with $\mathrm{Ga}^{+}$and $\mathrm{He}^{+}$at $30 \mathrm{keV}$.

dard Sn-ball sample for SEM calibration has been imaged with a $\mathrm{Ga}^{+}$FIB (Dual Beam Quanta 3d FEG from FEI) and with a $\mathrm{He}^{+}$FIB (ORION Plus from Zeiss SMT), and the evolution of two balls with similar initial diameter has been followed and plotted against the ion dose. [Since the sputter yield is a function of the incidence angle, a size effect is also present in the case of spherical features because for smaller balls the relative weight of the edges, more sensitive to sputter than flat surfaces, is higher; this effect is more prominent in the case of $\mathrm{He}^{+}$, for which the variation of the sputter yield for different angles of incidence is higher than for $\mathrm{Ga}^{+}$, see Fig. 3.] The imaging parameters for the two different experiments are listed in Table II. The graph also shows a linear fit, performed in order to estimate the rate of shrinking. [From the simulation the shrinking appears to be quadratic rather than linear, but in this case the features are followed only in the initial phase of the shrinking, where a linear fit appears to be adequate.] First of all, it appears that the dose required to fully disintegrate a ball does not differ much from the dose as predicted by the simulation. This is encouraging, meaning that our simple model can already give a good account of reality. More interesting is the relative rate of shrinking. For the $\mathrm{Ga}^{+}$, the features shrink with a rate of $1.3 \mathrm{~nm} / \mathrm{pC} \mu \mathrm{m}^{2}$, while for the $\mathrm{He}^{+}$the rate is $0.020 \mathrm{~nm} / \mathrm{pC} \mu \mathrm{m}^{2}$, leading to a ratio of $\sim 65$ : The same feature under $\mathrm{Ga}^{+}$will disappear 65 times faster than under $\mathrm{He}^{+}$bombardment, a value that is in good agreement with the simulation.

TABLE II. Imaging parameters for the Sn-ball sample imaged with the dual beam quanta $3 \mathrm{D}$ and with the ORION.

\begin{tabular}{lcc}
\hline \hline & Dual beam quanta 3D & ORION \\
\hline Calibration & 0.274 pixels $=1 \mathrm{~nm}$ & 2 pixels $=1 \mathrm{~nm}$ \\
Image size & $1024 \times 1024$ pixels & $1024 \times 1024$ pixels \\
Field of view & $3.65 \mu \mathrm{m}$ & $500 \mathrm{~nm}$ \\
Ion current & $1 \mathrm{pA}$ & $0.5 \mathrm{pA}$ \\
Scanning time & $\sim 90.5 \mathrm{~s} /$ frame & $\sim 100 \mathrm{~s} /$ frame \\
\hline
\end{tabular}

TABLE III. Imaging parameters for the three different sets of scans analyzed in this article.

\begin{tabular}{lc}
\hline \hline Imaging parameter & Value \\
\hline Image size & $1024 \times 1024$ pixels \\
Field of view & $500 \mathrm{~nm}$ \\
Ion current & $0.3-0.5 \mathrm{pA}$ \\
Ion energy & $\sim 26 \mathrm{keV}$ \\
Dwell time & $100,50,20,10$, and $5 \mu \mathrm{s}$ \\
\hline
\end{tabular}

\section{ON THE ACTUAL RESOLUTION THE HE MICROSCOPE}

In order to perform an analysis of the actual smallest detectable size of Sn balls in the He-SIM that takes into account also the effect of the beam on the sample, the procedure outlined in Sec. II, the details of which are explained in Ref. 8, has been used. We report here the formulas used to evaluate the $U_{I}$ and the $U_{S M}$,

$$
U_{I}=2 \frac{\sigma /\left|\tan \theta_{L}\right|+\sigma /\left|\tan \theta_{R}\right|}{\sqrt{2} \sqrt{\lfloor 0.2 D C\rfloor+1}},
$$

where $\sigma$ is the standard deviation of the shot noise in the image corrected for the intensity level of the feature being observed, $D$ is the ball's diameter, $C$ is the calibration factor in pixels $/ \mathrm{nm}$, and $\theta$ is the slope of the intensity profile evaluated at $50 \%$ of the step,

$$
U_{S M}=\int_{t^{*}-\Delta t / 2}^{t^{*}+\Delta t / 2} \frac{d y}{d t}(t) d t,
$$

which is just a way to express the size change of the feature between two scans; here, $y(t)$ is the mathematical expression of the curve that fits the evolution of the ball's diameter in time.

Sets of images have been acquired for five different dwell times, ranging from 100 down to $5 \mu \mathrm{s}$, with imaging parameters (image size, field of view, ion current, ion energy, and dwell time) summarized in Table III. The number of frames acquired for each set and the total imaging time are shown in Table IV. Figure 6 shows the first image for each set, from the longest dwell time to the shortest one: The difference in the level of noise is evident. Six images from the set acquired at $20 \mu$ s of dwell time are shown in Fig. 7, and it is

TABLE IV. Number of acquired frames and total imaging time for each set of images.

\begin{tabular}{cccc}
\hline \hline $\begin{array}{c}\text { Dwell time } \\
(\mu \mathrm{s})\end{array}$ & $\begin{array}{c}\text { Scan time } \\
(\mathrm{s})\end{array}$ & Number of frames & $\begin{array}{c}\text { Total scan time } \\
(\mathrm{s})\end{array}$ \\
\hline 100 & $\sim 105$ & 12 & $\sim 1260$ \\
50 & $\sim 52.5$ & 30 & $\sim 1575$ \\
20 & $\sim 21$ & 65 & $\sim 1365$ \\
10 & $\sim 10.5$ & 135 & $\sim 1417$ \\
5 & $\sim 5$ & 150 & $\sim 750$ \\
\hline
\end{tabular}




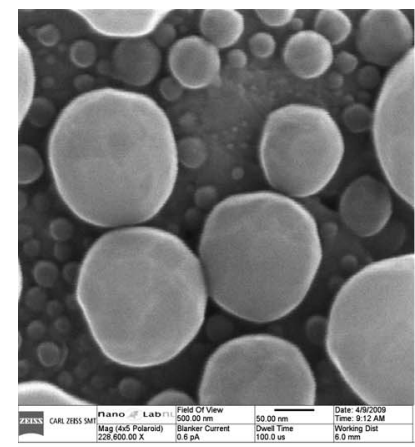

(a)

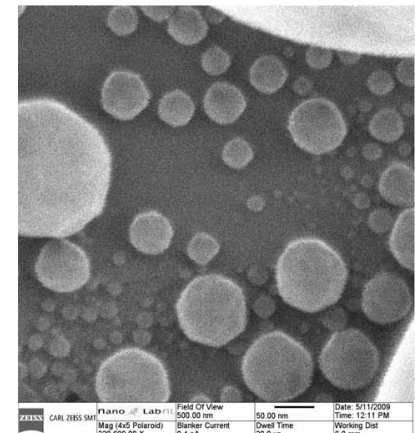

(c)

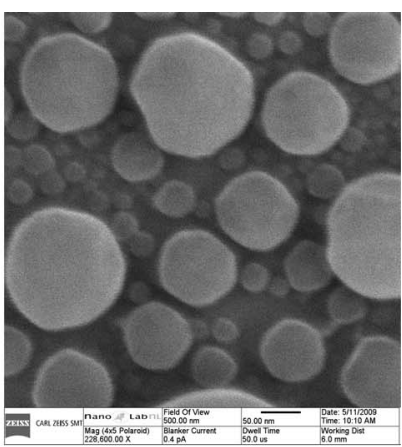

(b)

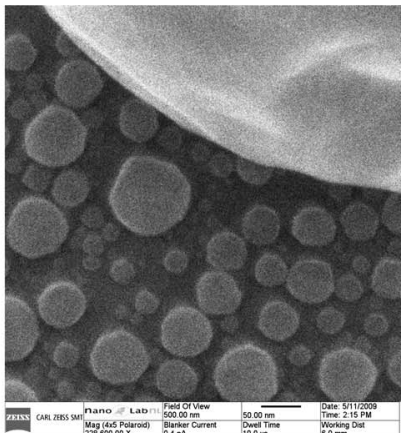

(d)

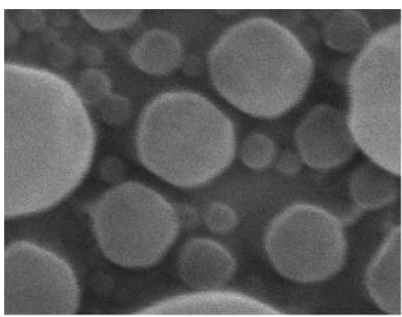

(a)

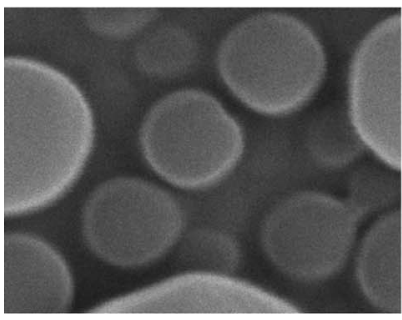

(c)

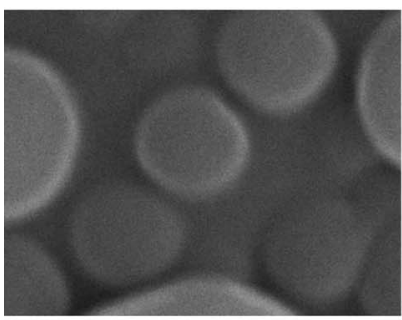

(e)

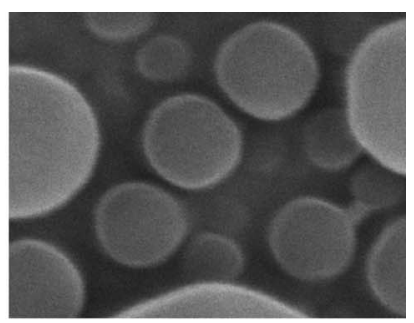

(b)

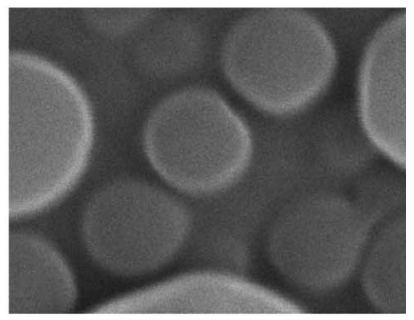

(d)

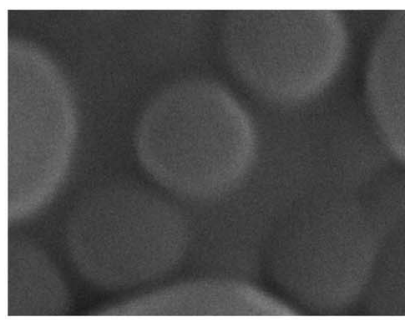

(f)

FIG. 7. Images from the series imaged with a dwell time of $50 \mu$ s, showing the dramatic effect on the sample of the $\mathrm{He}^{+}$at high magnification; here the field of view is slightly less than $500 \mathrm{~nm}$ because the images have been corrected for the shift; (a) after $\sim 52 \mathrm{~s}$ of imaging at $\sim 0.3 \mathrm{pA}$; (b) after $\sim 364 \mathrm{~s}$; (c) after $\sim 676 \mathrm{~s}$; (d) after $\sim 988 \mathrm{~s}$; (e) after $\sim 1300 \mathrm{~s}$; (f) after $\sim 1560$ s.

which can be easily calculated multiplying the sputtering sensitivity $S\left(\mu \mathrm{m}^{3} / \mathrm{nC}\right)$ for the dose rate $d_{r}\left(\mathrm{nC} / \mu \mathrm{m}^{2} \mathrm{~s}\right)$ and then for the scanning time $t_{\text {scan }}(\mathrm{s})$,

$$
U_{S}=S d_{r} t_{\mathrm{scan}}[=] \frac{\mu \mathrm{m}^{3}}{\mathrm{nC}} \frac{\mathrm{nC}}{\mu \mathrm{m}^{2} \mathrm{~s}} \mathrm{~s}=\mu \mathrm{m},
$$

immediately clear that for these extremely small fields of view, the effect of the $\mathrm{He}^{+}$on the sample cannot be neglected.

For each of the sets of images, the quantity $U_{I}$ has been calculated according to Eq. (4), for features of approximately $20 \mathrm{~nm}$ in diameter. The calculation of $U_{S M}$ has presented some difficulties because it turned out that the features did not shrink continuously, but they tended to fade once a typical size of $20 \mathrm{~nm}$ in diameter had been reached. For this reason, the amount of sputtering between two subsequent frames at the point of disappearance of the feature has been approximated by dividing the smallest diameter reached by the number of further frames that it took for the feature to disappear. The result of the analysis for the combination $\mathrm{He}^{+}-\mathrm{Sn}$ is plotted in Fig. 8, while Fig. 9 shows the same curves for $\mathrm{Ga}^{+}$on $\mathrm{Sn}$, for comparison. Both pictures also show the theoretical curves for $U_{S}$, as due to pure sputtering,

with $d_{r}=I_{\text {beam }} / A$, where $A$ is the area of the whole micrograph. Using for $S$ the values in Table I and referring to Table II for the ion current and the field of view, the following is found:

$$
U_{S}^{\mathrm{Ga}-\mathrm{Sn}}\left(t_{\mathrm{scan}}\right)=0.195 t_{\mathrm{scan}}[=] \mathrm{nm}
$$

and

$$
U_{S}^{\mathrm{He}-\mathrm{Sn}}\left(t_{\text {scan }}\right)=0.088 t_{\text {scan }}[=] \mathrm{nm} .
$$

Figure 8 also shows the values of the smallest visible feature for each set. This curve is included as a double check, as it takes into account both effects, uncertainty due to the collection of the secondaries and uncertainty due to the sputtering of target material. Here, the value of the smallest vis- 


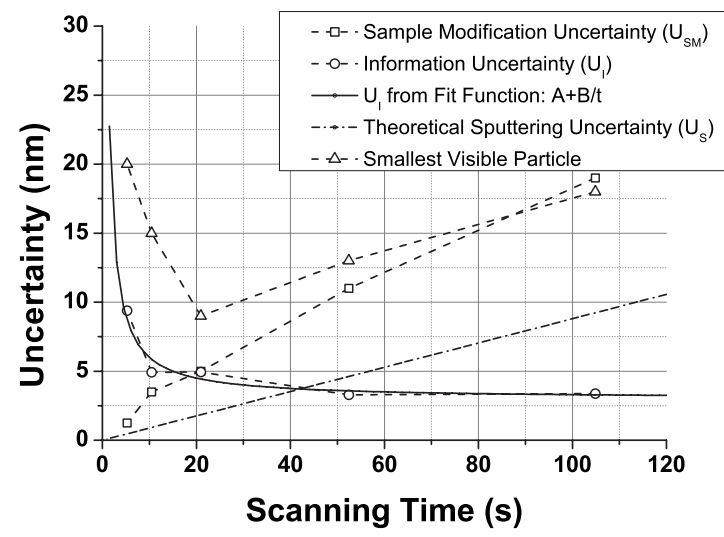

FIG. 8. $U_{I}, U_{S M}$, and $U_{S}$ plotted for different values of scanning time for 0.4 pA $\mathrm{He}^{+}$beam on $\mathrm{Sn}$; a plot of the smallest visible particle for each different scan is also present, which confirms that the best resolution $(\sim 5 \mathrm{~nm})$ is achieved for a dwell time of $20 \mu \mathrm{s}(\sim 20 \mathrm{~s}$ of scanning time for a 1024 $\times 1024$ micrograph). At this current and magnification, $100 \mathrm{~s}$ of scanning time correspond to a dose of $160 \mathrm{pC} / \mu \mathrm{m}^{2}$.

ible feature is rather arbitrary for it depends on the threshold adopted in the definition of feature itself, but the fact that it shows a minimum for the same value of scan time for which the curves $U_{I}$ and $U_{S M}$ intersect is a good indication that the best value of scan time can be trusted.

Comparing Figs. 8 and 9, a few interesting observations can be made.

- The actual resolution for the Sn-ball sample found for the He-SIM is $\sim 5 \mathrm{~nm}$, almost five times better than what found for the Ga-scanning ion microscopy $(\sim 23 \mathrm{~nm})$. In both cases the value is set by the beam/sample interaction. The observed limit of $5 \mathrm{~nm}$ is of course linked to the sample used, both in terms of geometry and in terms of materials, and it could be lowered in special cases (flat geometries, samples with a lower sputter sensitivity).

- Comparing the two curves of $U_{S M}$ and $U_{S}$ versus scan time, it appears that the two curves basically coincide in the case of the $\mathrm{Ga}^{+}$beam, while for the $\mathrm{He}^{+}$beam the observed $U_{S M}$ is twice the calculated $U_{S}$; this translates in an experimental ratio of the sputtering rate between $\mathrm{Ga}^{+}$ and $\mathrm{He}^{+}$of $\sim 30$, about half of the theoretical ratio.

- The lower sputtering is not the only advantage of the HeSIM, which exhibits very low values of the parameter $U_{I}$; recalling Eq. (4), it can be seen that $U_{I}$ takes into account three different effects: the shot noise in the image that is connected with the detection of secondary electrons, the $a$ priori knowledge on the sample, and the steepness of the edges in the intensity profile; the low values of $U_{I}$ for the He-SIM have to be ascribed to the fact that the probe size is extremely small compared to the Ga-SIM $(\sim 0.3 \mathrm{~nm}$ for the ORION, $\sim 5 \mathrm{~nm}$ for the Ga FIB).

- Further investigation is required in order to understand and quantify the role of the local redeposition and the redeposition of atoms from different areas of the sample (contamination).

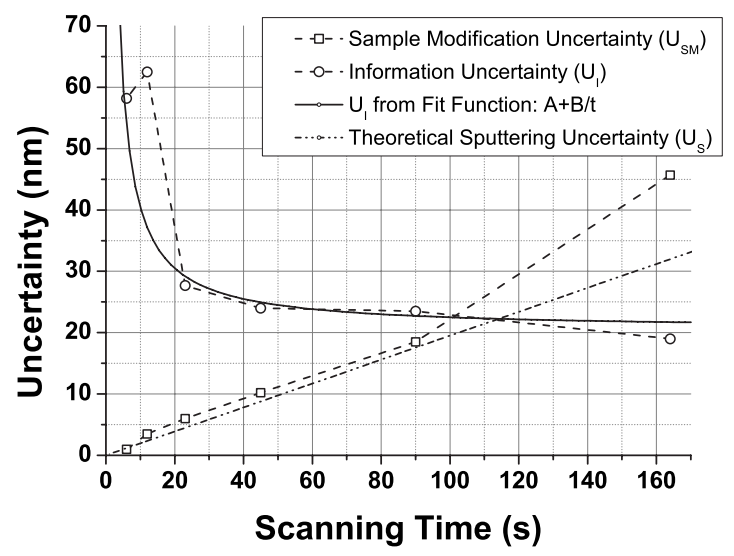

FIG. 9. $U_{I}, U_{S M}$, and $U_{S}$ plotted for different values of scanning time for 1 pA Ga ${ }^{+}$beam on $\mathrm{Sn}$, showing that the best resolution $(\sim 23 \mathrm{~nm})$ is achieved for a scanning time of $\sim 100 \mathrm{~s}$ (corresponding to $\sim 100 \mu \mathrm{s}$ dwell time for a $1024 \times 1024$ micrograph). At this current and magnification, $100 \mathrm{~s}$ of scanning time correspond to a dose of $7.5 \mathrm{pC} / \mu \mathrm{m}^{2}$.

\section{CONCLUSIONS}

The main conclusions of this article are here summarized.

- Image resolution in SIM is a dynamic concept: At present the concept of resolution is presented as static in the sense that it is calculated based either on the optical system properties (probe size), or on the characteristics of a single micrograph (edge resolution, gap resolution, and inverse of the highest spatial frequency); this is not applicable when sample modification plays a role, in which case a series of images is required in order to quantify the effect of the beam on the target.

- Image resolution is not a property of the optical system only: There is no case in which a single number can be assigned to a machine to characterize its performance, which is the result of the interaction between ions and sample; as a consequence, the system to be analyzed is not only the microscope itself, but the combination of the microscope and the sample, both in terms of materials (different atom species are differently affected by the impinging ions) and geometry (even for the same material, features on the sample undergo different modifications if, for example, they are flat or their size is different in different layers).

- Accuracy of measurement and smallest detectable feature are two different concepts: In particular the first one can be made independent of the sputtering effect since this increases monotonically with the scanning time and can be modeled and quantified, while the second is intrinsically connected with the sample modification.

To support the concept of dynamic resolution, the procedure that has been proposed and defined in Ref. 8 has been applied to the combination He-SIM/Sn-ball sample, which led to interesting results. In particular, it has been shown that as expected, the performance (in terms of smallest detectable feature) of the He-SIM is better than the traditional Ga-SIM by a factor of, at least, 5 . 


\section{ACKNOWLEDGMENTS}

This work is part of the research program "Microscopy and Modification of Nano-structures with focused electron and ion beams" (MMN) of the "Stichting voor Fundamenteel Onderzoek der Materie" (FOM), which is financially supported by the "Nederlandse Organisatie voor Wetenschappelijk Onderzoek" (NWO). The MMN program is cofinanced by FEI Co. In addition, support of the BSIK NanoNed program of the Dutch Ministry of Economic Affairs is gratefully acknowledged.
${ }^{1}$ A. Vladár, M. Postek, and B. Ming, Microscopy Today 17, 2 (2009).

${ }^{2}$ E. Kieft and E. Bosch, J. Phys. D: Appl. Phys. 41, 215310 (2008).

${ }^{3}$ J. Orloff, L. W. Swanson, and M. Utlaut, J. Vac. Sci. Technol. B 14, 3759 (1996).

${ }^{4}$ M. Sato, in Handbook of Charged Particle Optics, edited by J. Orloff (CRC, Boca Raton, FL, 2009), Chap. 8, pp. 391-436.

${ }^{5}$ M. Sato and J. Orloff, Ultramicroscopy 41, 181 (1992).

${ }^{6}$ V. Castaldo, C. W. Hagen, B. Rieger, and P. Kruit, J. Vac. Sci. Technol. B 26, 2107 (2008)

${ }^{7}$ J. Orloff, Proc. SPIE 2522, 412 (1995).

${ }^{8}$ SRIM/TRIM reference website: http://www.srim.org/. 